Trauma Berufskrankh 2004 - 6 [Suppl 3] : S357-S367 DOI 10.1007/s10039-004-0899-0

Online publiziert: 24. Juni 2004

C) Springer-Verlag 2004

J.-D. Rompe · C. Schöllner · J. Heine • Orthopädische Universitätsklinik, Mainz

\title{
Operative und \\ nichtoperative Behandlung bei Pseudarthrosen
}

\section{Hochenergetische Stoßwellentherapie}

gen Röhrenknochen als Zeichen eines übergeordneten systemischen

Regulationssystems einhergeht

Nach Perren u. Cordey [54] bedeutet Knochen- oder Frakturheilung die biologische Stabilisierung des Knochens, der seine Stützfunktion nicht mehr erfüllt. Sie besteht in der Überwindung der pathologischen Beweglichkeit im Frakturspalt und in der Wiederherstellung kraftschlüssiger Formsteifigkeit durch Knochenneubildung. McKibbin [48] wies darauf hin, dass der Ablauf einer Knochenheilung definitionsgemäß einer Knochenregeneration entspricht, da keine minderwertige Narbe die verletzten Strukturen ersetzt, sondern normales, dem ursprünglichen Gewebe entsprechendes Regenerat. Hulth [36], Wolff [84], Pennig [51], Draenert [20], Perren [53], Braun u. Rüter [10] und Stürmer [72] haben in ihren Übersichtsarbeiten die Konzepte der Frakturheilung ausführlich dargelegt.

Als Voraussetzung für den regelrechten Ablauf der Knochenheilung nannten Braun u. Rüter [10] 2 Faktoren:

1. eine regionale Steigerung des physiologischen Knochenumbaus (regionales akzeleratorisches Phänomen), die Frost [27] als Hinweis auf einen lokalen Steuerungsmechanismus der Frakturheilung ansah

2. ein parallel nachweisbares „systemisches akzeleratorisches Phänomen“, das nach Müller et al. [49] mit einer frakturassoziierten Zunahme der Knochenmasse im spongiosen Knochen des Stammskeletts und der lan-
Die zugrunde liegenden Zellleistungen können über die Wege der Modulation und Induktion ausgelöst werden. Als mögliche Modulatoren und Induktoren konnten im Frakturkallus folgende Faktoren nachgewiesen werden:

- Zelldebris

- mechanische Einflüsse

- Qualität der Blutversorgung und Sauerstoffsättigung

- Bioelektrizität

- humorale Faktoren

\section{Gestörte Knochenheilung}

Sie ist nachhaltig gestört

- bei einer fehlenden medullaren oder periostalen Gefäßversorgung,

- bei einer Instabilität zum falschen Zeitpunkt oder

- bei einem mangelnden Fragmentkontakt.

Fehlende medullare oder periostale Gefäßversorgung

Jede Fraktur führt zu einer Knochennekrose der unmittelbaren Bruchzone. Stückfrakturen unterbrechen die medullare Gefäßversorgung, ebenso kann sie durch das Aufbohren der Markhöhle bei der Marknagelung in Mitleidenschaft gezogen werden. Dislozierte Frakturen mit gedecktem oder offenem Weichteilschaden gehen mit einer Unterbrechung der periostalen Durchblutung einher. Prinzipiell können Kortikalisnekrosen bei der spontanen Knochenheilung durch periostale Kallusspangen überbrückt werden. Haben die Nekrosen wieder Gefäßanschluss gefunden, ist eine langsame Vaskularisation durch Havers-Umbau möglich. Wenn das Periost geschädigt ist, wird die Kallusbildung inkomplett sein. Nekrotische Fragmente werden dann aseptisch sequestrieren.

\section{Instabilität zum falschen Zeitpunkt}

Eine Störung der Knochenheilung wird auch bei lokaler Überlastung des Knochens in der vulnerablen Phase, d. h. bei Überbrückung des Frakturspalts durch Faserknochen, beobachtet. Der vorhandene Kallus hat sich zwischenzeitlich in seinem Elastizitätsverhalten der Kortikalis angenähert und kann eine neu aufgetretene Instabilität nicht mehr abfedern: Die zarten Knochenbälkchen brechen, die spontane Knochenheilung muss von vorn beginnen. Falls keine ausreichende Kallusvermehrung oder Fragmentresorption mehr möglich sind, kommt es zur Pseudarthrose.

\section{Mangelnder Fragmentkontakt}

Er führt bei der spontanen Knochenheilung nur selten zu Störungen. Eine direkte 
Knochenheilung ist ohne ihn nicht möglich. Bei einer absolut stabilen Osteosynthese über einem Defekt kann infolge des fehlenden Instabilitätsreizes auch kein periostaler oder endostaler Kallus gebildet werden, sodass eine Überbrückung des Defekts auch im Rahmen der spontanen Knochenheilung nicht stattfinden wird. Folge sind entweder eine inkomplette Heilung in den Kontaktbereichen oder eine Pseudarthrose mit nachfolgender Auslockerung der Implantate $[36,39,63,72]$.

\section{Klassifikation der Pseudarthrosen}

Allgemein anerkannt und in der Therapieplanung bestens bewährt ist die Einteilung nach Weber u. Cech [8o]. Sie unterschieden aktive von inaktiven Pseudarthrosen, wobei die morphologische Einteilung die noch vorhandene osteogenetische Aktivität widerspiegelt [79]. Nach wie vor kann lediglich die Szintigraphie die aktuelle Osteogenese semiquantitativ ermitteln. Erst hierdurch wird die Unterscheidung in aktive oder inaktive Pseudarthrosen möglich [69].

Zu den stark Nuklid anreichernden aktiven Pseudarthrosen gehören:

1. biologisch reaktionsfähiger Typ Typ A1 (vital, kallusreich, hypertroph, Elefantenfußpseudarthrose)

2. biologisch weniger reaktionsfähiger Typ - Typ A2 (vital, kallusarm, Pferdefußpseudarthrose)

3. biologisch reaktionsloser Typ Typ A3 (oligotroph, kalluslos)

Von den aktiven werden die biologisch reaktionsunfähigen bzw. avitalen inaktiven Pseudarthrosen unterschieden:

1. Drehkeilpseudarthrose - Typ B1 (dystroph)

2. Defektpseudarthrose - Typ B2

(fehlende Knochensubstanz)

3. atrophe Pseudarthrose - Тур B3 (Narbengewebe)

\section{Operative Maßnahmen}

In der Frakturbehandlung ist die Anzahl aufgetretener Knochenheilungsstörungen trotz verbesserter Behandlungskonzepte und Verwendung verfeinerter Osteo- syntheseverfahren mit etwa $5 \%$ auf einem konstanten Niveau geblieben $[6,45]$. Auch im Rahmen elektiver Operationen am Skelett zur Korrektur z. B. von Achsenfehlstellungen stellt die Pseudarthrosenbildung mit einer Häufigkeit von etwa 1-2\% eine gefürchtete Komplikation und therapeutische Herausforderung dar.

In der Therapie von Pseudarthrosen sind chirurgische Verfahren nicht zuletzt durch die Einführung verbesserter Osteosynthesematerialien wie Marknagel [43], Ringfixateur [38] und unilateraler Fixateur [16] zum „Goldstandard“ geworden. Allgemein ist Behandlungsziel die Konsolidierung der Pseudarthrose zusammen mit der Beseitigung von Fehlstellungen und Längenunterschieden sowie der Berücksichtigung von Weichteilschäden.

Bei der Behandlung von hypertrophen Pseudarthrosen geht es im Wesentlichen allein um die Herstellung von Stabilität. Ein Anfrischen oder gar Resezieren des Pseudarthrosengewebes ist unnötig, ebenso eine Spongiosaanlagerung.

Im Fall einer atrophen Pseudarthrose liegen in der Regel eine schwer gestörte lokale Perfusion und/oder ein knöcherner Defekt vor. Operativ steht die Anfrischung der noch durchbluteten Hauptfragmente im Vordergrund. Zusätzlich empfahlen Kuner et al. [45] die Dekortikation über 5-6 cm am proximalen und distalen Hauptfragment sowie um die halbe Zirkumferenz herum, wobei die Weichteile fest mit der abgemeißelten Kortikalisschicht im Verband bleiben müssen.

Defektpseudarthrosen bis $\mathrm{zu} 1,5 \mathrm{~cm}$ können durch osteoplastische Maßnahmen zusammen mit Achsenkorrektur behandelt werden. Bei größeren Distanzen sollte neben der knöchernen Konsolidierung auch die Wiederherstellung der anatomischen Länge angestrebt werden. Folgende Maßnahmen stehen hier zur Verfügung:

- Anfrischen, Dekortikation, autologe Spanverschraubung und Spongiosaplastik, Stabilisierung durch Osteosynthese

- Resektion des Defekts, Verkürzung und Osteosynthese, Längenwiederherstellung mittels Kallusdistraktion - Fibula-pro-Tibia-Operation mit zusätzlicher passagerer Stabilisierung mit Fixateur externe
Der zeitliche Ablauf erstreckt sich in jedem Fall über viele Monate. Pseudarthrosen des Humerus konnten von Cattaneo et al. [13] mit dem Ilisarov-Apparat in $86 \%$ zur Ausheilung gebracht werden. Die Behandlung von Femurpseudarthrosen mit Marknägeln durch Wu et al. [85] war in $88 \%$ erfolgreich. Bei tibialer Lokalisation führte die Verwendung von Knochentransplantaten allein in 85-93\%, die Revision mittels Marknagel in 89-93\% der Fälle zum Erfolg $[8,61,78,83]$.

\section{Nichtoperative Maßnahmen}

Trotz der oben angeführten Erfolgsquoten dürfen die Probleme des chirurgischen Vorgehens (Narkoserisiko, allgemeines Operationsrisiko, Materialversagen usw.) nicht außer Acht gelassen werden: Schwierigkeiten bei der evtl. notwendigen autogenen Knochentransplantation ergeben sich durch lokale Komplikationen (z. B. Weichteilinfekt, Hämatom, Verletzung benachbarter Nerven) an der Entnahmestelle als so genannte ,donor site morbidity" mit einer Häufigkeit von 6-20\% [15, 89].

Dass Pseudarthrosenbehandlung und Osteosynthese im deutschsprachigen Raum heute weitgehend gleichgesetzt werden, wird an einer 100-seitigen, von Tscherne herausgegebenen Übersicht verschiedener Arbeitsgruppen in der Zeitschrift „Der Orthopäde“ im Oktober 1996 deutlich.Lediglich auf 1 1/2 Seiten wurden die Möglichkeiten nichtoperativer Verfahren abgehandelt. Die Frage „Gibt es neben der lokalen chirurgischen Frakturbehandlung weitere gesicherte Möglichkeiten der positiven Beeinflussung der Bruchheilung?" beantworteten Braun u. Rüter [10] eindeutig mit nein.

Zunächst sollen die aktuellen Forschungsergebnisse der von Einhorn [22] als biophysikalische Maßnahmen bezeichneten elektrischen und Ultraschallstimulation auf die verzögerte Knochenheilung vorgestellt werden. Anschließend werden unsere Erfahrungen mit der extrakorporalen Stoßwellentherapie geschildert.

\section{Elektrostimulation}

Ihr wird seit den ersten Arbeiten von Yasuda [87] sowie Fukada u. Yasuda [28] ein 
positiver Effekt auf die Knochenheilung zugeschrieben. Technisch sind heute im Wesentlichen 3 Formen zu unterscheiden:

1. Die direkte Strombehandlung unter Benutzung implantierter Elektroden als invasives Verfahren (20 mA Stromstärke/24 h/12 Wochen)

2. Die Behandlung im Kondensatorfeld (6o kHz Wechselspannung, 5 V Amplitude/24 h/12 Wochen)

3. Die Behandlung im gepulsten Magnetfeld (symmetrische, rechteckige elektrische Impulse, Pulsbreite 5 ms, Pulsrepetitionsfrequenz $15 \mathrm{~Hz}$, Pulsamplitude $20-140 \mathrm{mV}$, Signalfrequenz $70 \mathrm{kHz} / 8 \mathrm{~h}-24 \mathrm{~h} / 12$ Wochen)

\section{Biologische Grundlagen}

Bekannt ist, dass bei der Biegung eines Knochens negative und positive Ladungen getrennt werden. Dabei ist der negative Pol auf der komprimierten, konkaven, der positive Pol auf der konvexen Seite zu finden. Diese belastungsinduzierten Potenziale gehen von den anorganischen, mineralischen Bestandteilen des Knochens aus und können unabhängig vom Vorhandensein vitalen Gewebes erzeugt werden. Sie sind deswegen wahrscheinlich auf piezoelektrische Effekte zurückzuführen [3]. Demgegenüber finden sich elektronegative bioelektrische Potenziale in Gebieten aktiven Knochenwachstums und -umbaus, elektropositive in biologisch inaktiven $\mathrm{Zo}$ nen.

Friedenberg u. Brighton [25] wiesen zusätzlich an unbelastetem Knochen ein elektronegatives Steady-state-Potenzial nach.

\section{Literatur}

Einzelerfolge. 1971 berichteten Friedenberg et al. [26] in einem Fallbericht über die Heilung einer Pseudarthrose des medialen Malleolus nach Stromapplikation über in den Knochen implantierte Elektroden. Kraus u. Lechner [41] beschrieben 1973 erstmalig die Regeneration von Knochengewebe nach Stimulation durch elektromagnetische Potenziale. Ihre Methode beruhte auf 2 Komponenten, nämlich einem alternierenden Magnetfeld und einer induzierten Wechselspannung über operativ implantierte Elektroden.
1974 erzielten Bassett et al. [4] mit einer alleinigen Magnetfeldtherapie ebenfalls Einzelerfolge in der Pseudarthrosenbehandlung.

Indikation für diese nichtoperativen Behandlungsformen waren typischerweise konservativ im Gips behandelbare aktive Pseudarthrosen der Typen A1 - vital, kallusreich, hypertroph - bis A3 - oligotroph, kalluslos - nach der Klassifikation von Weber u. Cech [80].

Wirkmechanismus. Diesen erklärte Kuner [44] damit, dass die niederfrequente Wechselstromwirkung die Imitation physiologisch auftretender biphasischer Potenziale mit Beeinflussung des Zellstoffwechsels durch Überwindung des zelleigenen Membranpotenzials ermöglicht. Nach Lechner [46] ist allerdings Voraussetzung, dass physiologische Leitungsbedingungen vorliegen. Deperiostierter oder devaskularisierter Knochen sowie instabile Frakturen haben so hohe elektrische Widerstände, dass eine Elektrostimulation über die physiologische Knochenheilung nicht möglich ist.

Experimentelle Unersuchungen. Blümlein et al. [9] und Stürmer et al. [73] überprüften tierexperimentell die Ergebnisse von Kraus u. Lechner [41]. Bei qualitativer und quantitativer Auswertung von Röntgenbildern und Histologie des unentkalkten Knochens fanden sich keine Unterschiede zur Kontrollgruppe. Erst die Kombination von Magnetfeld und Spongiosaplastik zeigte ein besseres Ergebnis. Dies konnte Schmit-Neuerburg [66] 1980 bestätigen. Ascherl [1] erklärte diesen positiven Effekt dadurch, dass Spongiosa im Gegensatz zur Kompakta einen sehr niedrigen Widerstand hat. Durch die Fragmentanfrischung und eine Spongiosaplastik werden optimale Bedingungen geschaffen, die zu einer Verbesserung der Leitfähigkeit bis zum 4oFachen führen.

1977 gelang es Zichner [91] bei Osteotomiemodellen der Hundeulna nicht, einen positiven Effekt der direkten Stromapplikation im Vergleich zu einer Kontrollgruppe nachzuweisen. Er beschrieb aber eine 25\%ige Verkürzung der Heilungszeit von Pseudarthrosen.

Weder das Kombinationsverfahren mit niederfrequentem Magnetfeld und indu-
Trauma Berufskrankh

$2004 \cdot 6$ [Suppl 3] : S357-S367

DOI 10.1007/s10039-004-0899-0

(C) Springer-Verlag 2004

J.-D. Rompe $\cdot$ C. Schöllner $\cdot$ J. Heine

Operative und nichtoperative Behandlung bei Pseudarthrosen. Hochenergetische Stoßwellentherapie

\section{Zusammenfassung}

In diesem Übersichtsartikel werden der physiologische und der gestörte Knochenheilungsprozess beleuchtet. Sämtliche operativen und nicht operativen Behandlungsverfahren basieren auf klinischer Empirie, erfüllen aber nicht die Kriterien der evidenzbasierten Medizin. Vor diesem Hintergrund kann die hochenergetische Stoßwellentherapie bei ausgewählten, erfolglos voroperierten Patienten durchaus eine Behandlungsalternative darstellen. Sie sollte aber nach wie vor ausschließlich im Rahmen klinischer Studien zum Einsatz kommen.

\section{Schlüsselwörter}

Knochenheilung · Pseudarthrose

Stoßwellentherapie

\section{Surgical and nonsurgical treat- ment of pseudarthroses. High- energy shockwave therapy}

\section{Abstract}

This review article summarizes our present knowledge on the physiological and on impaired processes in bone healing. All the clinical procedures applied are based on empirical clinical data and do not qualify as evidencebased medicine. Against this backdrop, highenergy shockwave treatment may well be a treatment alternative in selected patients who have already undergone surgery but are still suffering from a pseudarthrosis. For the time being, however, this form of therapy should be used only in clinical studies.

\section{Keywords}

Bone healing · Pseudarthrosis - Shockwave treatment 


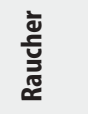

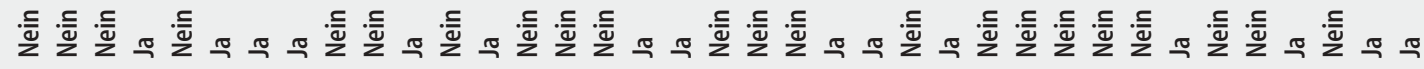

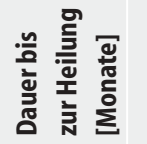

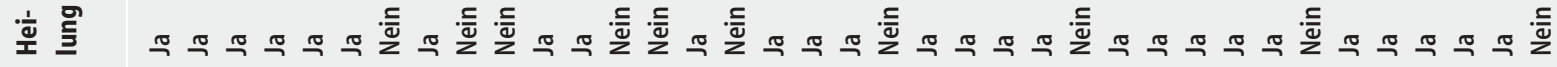

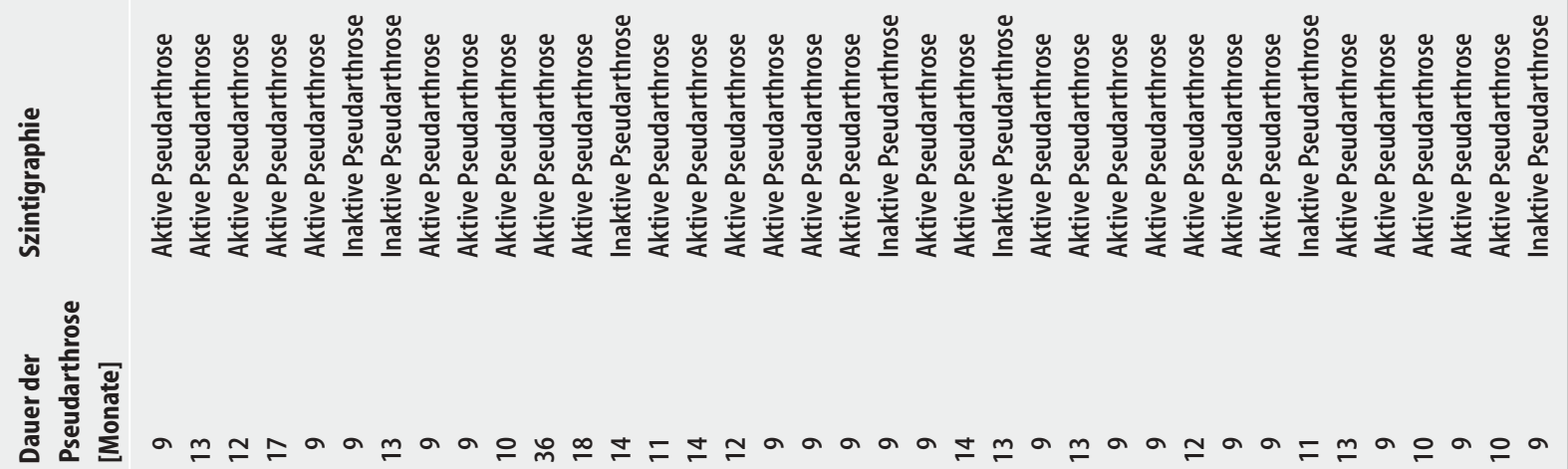

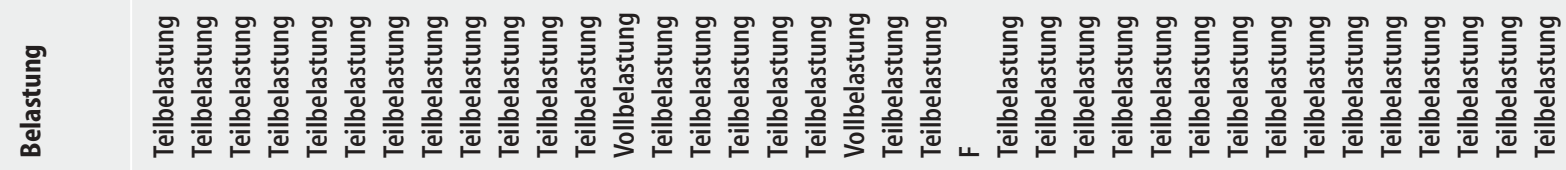

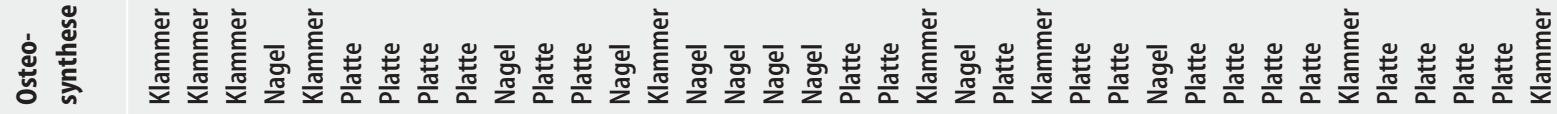
高 ํํㅇ 흘

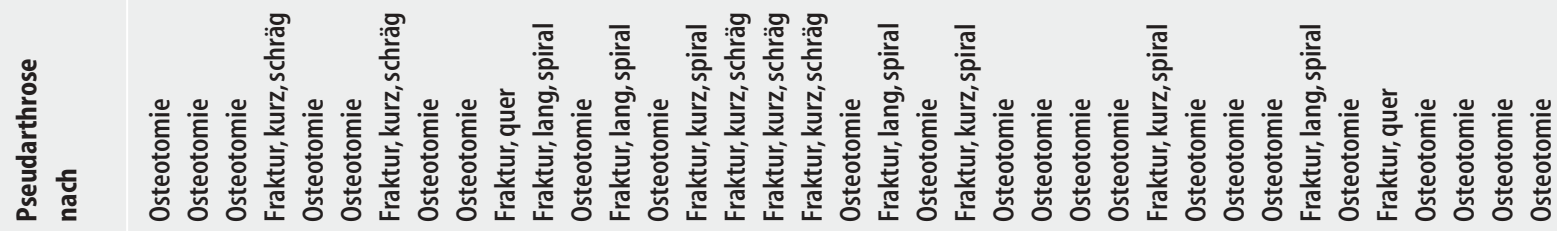

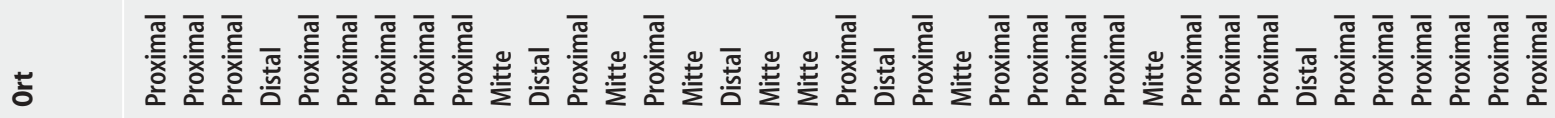

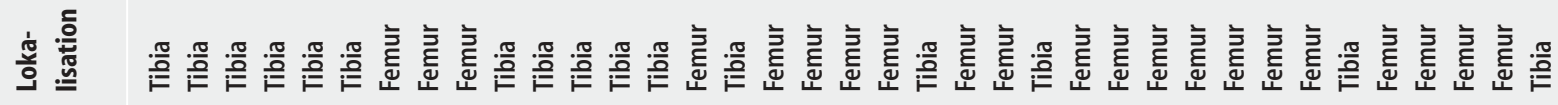

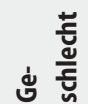

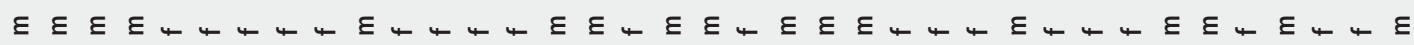

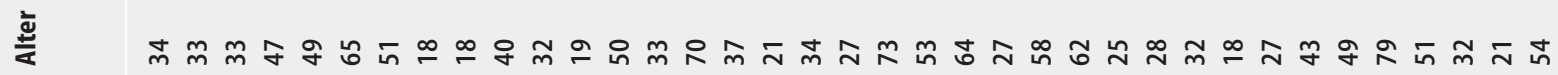




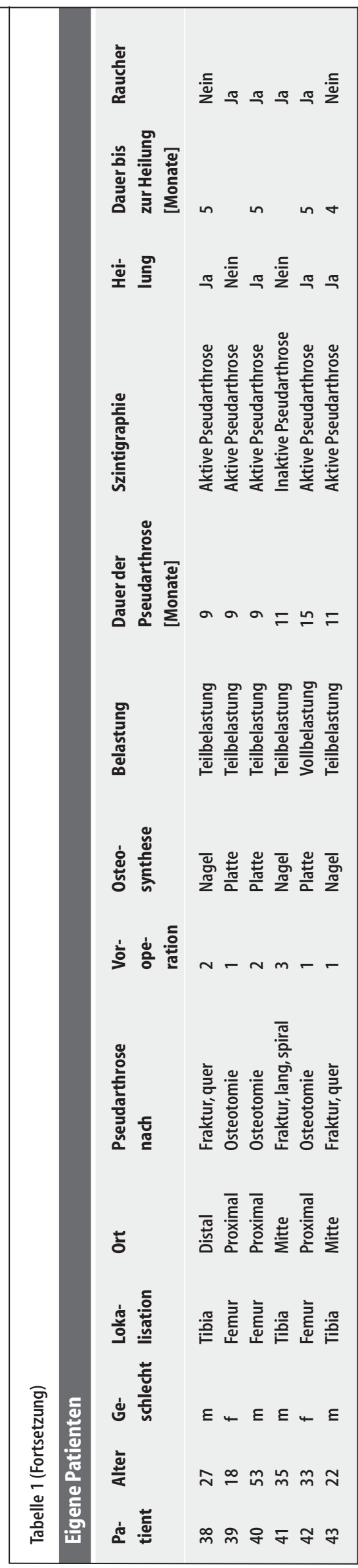

zierter Wechselspannung noch die direkte Stromapplikation fanden im deutschen Sprachraum Verbreitung. Kuner et al. [45] betonten dazu, dass mit Anfrischung der Pseudarthrosenränder, Spongiosaplastik und Osteosynthese mit Implantaten mit großer Sicherheit alle vaskularisierten Pseudarthrosen zu einer knöchernen Konsolidierung zu bringen sind.

Von angloamerikanischen Arbeitsgruppen wurde dem nichtinvasiven Therapieansatz mehr Beachtung geschenkt.

Auf tierexperimenteller Seite konnten Pienkowski et al. [55] in einer randomisierten Doppelblindstudie an 399 frisch osteotomierten Kaninchenfibulae einen signifikanten Anstieg der Kallusfestigkeit nach Magnetfeldtherapie nachweisen.

Deibert et al. [17] zeigten bei dem gleichen Modell, dass die Steifheit des Kallus mit der täglichen Applikationszeit korrelierte, wobei die besten Ergebnisse bei 24-stündiger Anwendung gefunden wurden.

Rijal et al. [57] beschrieben anhand einer verzögerten Bruchheilung des Kaninchenradius einen positiven Einfluss des Kondensatorfelds auf Kallusformation und Knochendichte. Dabei wird angenommen, dass es durch das elektrische Wechselfeld - entsprechend einer zyklischen Belastung - zur Ausrichtung von Kollagenfibrillen zu Faserbündeln mit konsekutiv vermehrter Mineralisation dieser Bündel kommt.

Pepper et al. [52] wiesen allerdings nach tibialen Verlängerungsoperationen in Ilisarov-Technik bei Hunden nach Anlage eines Kondensatorfelds einen negativen Effekt auf das Knochenregenerat bezüglich Bruch- und Verwindungsfestigkeit nach.

Klinische Untersuchungen. Klinisch lagen zunächst nur prospektive, nicht kontrollierte Untersuchungen vor. So stellten Bassett et al. [5] eine Multicenterstudie von 127 diaphysären Tibiapseudarthrosen nach elektromagnetischer Stimulation vor, wobei eine Durchbaurate von $87 \%$ erzielt wurde. Eine Kontrollgruppe fehlte.

Conolly [14] berichtete nach direkter Stromapplikation über intramedullar implantierte Elektroden über einen Heilungserfolg bei 32 von 44 Pseudarthrosen des Femurs und der Tibia innerhalb eines 6-monatigen Zeitraums.
Barker et al. [2] veröffentlichten 1984 eine Doppelblindstudie über die unterschiedlich starke Magnetfeldbehandlung bei 16 Patienten mit einer Tibiapseudarthrose. Ein Unterschied der beiden Gruppen wurde nicht gefunden.

Sharrard [70] präsentierte 20 Patienten mit einer verzögerten Knochenheilung, also keiner Pseudarthrose, in einer doppelblind randomisierten Studie. Nach Magnetfeldtherapie kam es zu einer knöchernen Konsolidierung bei 9 von 20 Patienten. Die Kontrollgruppe umfasste $25 \mathrm{~Pa}-$ tienten, wobei lediglich 3-mal eine Heilung beobachtet wurde.

Parnell u. Simonis [50] wiesen in einer kontrollierten Studie einen signifikanten Effekt im gepulsten Magnetfeld an $34 \mathrm{~Pa}$ tienten mit einer Tibiapseudarthrose nach.

Scott u. King [67] präsentierten vor 2 Jahren eine prospektive, randomisierte, doppelblinde Studie über die Kondensatorfeldbehandlung an 23 Patienten mit Pseudarthrosen verschiedener langer Röhrenknochen. 21 Patienten beendeten die Untersuchung nach 6 Monaten. Die Pseudarthrose heilte in 6 von 10 Fällen nach Elektrostimulation aus, aber bei keinem der 11 mit Plazebo behandelten Patienten. Dieser Unterschied war hochsignifikant.

Zamora-Navas et al. [90] behandelten ebenfalls im Kondensatorfeld und erzielten bei 22 Patienten 16-mal eine knöcherne Konsolidierung.

In einer retrospektiven, statistisch sehr sorgfältig überprüften Zusammenfassung von 271 seit 1971 behandelten Tibiapseudarthrosen kamen Brighton et al. [11] zu dem Ergebnis, dass bei aktiven vitalen Pseudarthrosen nach unkomplizierten Typ-A-Frakturen die Spongiosaplastik mit einer Erfolgswahrscheinlichkeit von 99\% nicht besser abschnitt als die direkte Stromapplikation (99\%) oder die Behandlung im Kondensatorfeld (96\%), kurz, dass bei diesen prognostisch günstigen Knochenheilungsstörungen ein operativer Reeingriff durch ein nichtinvasives Verfahren ersetzt werden kann.

Die Elektrostimulation hat aber 2 wesentliche Nachteile:

1. Die direkte Stromapplikation kann nur nach operativer Elektrodenimplantation durchgeführt werden, was 
für den Patienten ein erhöhtes Infektionsrisiko bedeutet.

2. Die Behandlung im Kondensatoroder elektromagnetischen Feld muss über mehrere Monate mindestens $12 \mathrm{~h}$ am Tag durchgeführt werden.

\section{Niedrigenergetischer gepulster Ultraschall}

\section{Experimentelle Befunde}

Anfang der 8oer Jahre berichtete Duarte [21] erstmalig über eine Stimulation der Knochenheilung am Kaninchenosteotomiemodell mittels niedrigenergetischem Ultraschall. Die Schallenergie lag bei dieser therapeutischen Anwendung bei $50 \mathrm{~mW} / \mathrm{cm}^{2}$ und damit im Bereich des diagnostischen Ultraschalls. Mit einer Repetitionsrate von $1000 \mathrm{~Hz}$ wurden $5 \mu \mathrm{s}$ lange Ultraschallpulse über 15 min täglich appliziert. Bereits eine 9-tägige Behandlung reichte aus, um die Kontrollgruppe qualitativ und quantitativ bezüglich der Kallusbildung zu übertreffen. Der Autor führte den Effekt auf die piezoelektrische Stimulation des Knochens durch den Ultraschall zurück. Kavitations- oder thermale Effekte wurden nicht beobachtet.

Pilla et al. [56] fanden am gleichen Modell heraus, dass nach 14- bis 23-tägiger Ultraschallbehandlung (jeweils $20 \mathrm{~min} /$ Tag) die behandelten Fibulae sich im Torsionstest deutlich fester zeigten als die nichtultraschallbehandelte Kontrollgruppe. Nach 4 Wochen war in beiden Gruppen eine vergleichbare Torsionsfestigkeit anzutreffen.

Wang et al. [77] behandelten Femurschaftfrakturen bei Ratten über 14 Tage postoperativ mit niedrigenergetischem Ultraschall. Appliziert wurden $200 \mu$ s lange Pulse (Sinuswellen 1,5 MHz) mit einer Repetitionsfrequenz von $1 \mathrm{kHz}$. Die Anwendungsdauer betrug $15 \mathrm{~min}$ täglich. Von 16 ultraschallbehandelten Frakturen durchbauten 5 , von 28 nichtbehandelten keine innerhalb von 3 Wochen.

Bis heute liegen keine tierexperimentellen Untersuchungen über die Möglichkeit der Pseudarthrosenbehandlung vor.

\section{Klinische Ergebnisse}

Auf klinischer Seite stellten Xavier u. Duarte [86] Fallbeispiele der Pseudarthrosenbehandlung mittels Ultraschall vor und gaben eine Erfolgsquote von $84 \%$ bei Tibia- und von $85 \%$ bei Femurpseudarthrosen an.

1994 präsentierten Heckman et al. [35] eine prospektive, kontrollierte Doppelblindstudie, wobei Ultraschall als Ergänzung einer Gipsruhigstellung bei Typ-ITibiafrakturen zur Anwendung kam. 33 Frakturen der Therapiegruppe waren nach durchschnittlich 96 Tagen konsolidiert im Vergleich zu 34 Frakturen der Plazebogruppe, bei denen eine Heilung nach durchschnittlich 154 Tagen beobachtet wurde.

1996 fassten Frankel et al. [24] 500 behandelte Pseudarthrosen zusammen und gaben eine Erfolgsquote von $89 \%$ an bei einer durchschnittlichen Behandlungsdauer von 114 Tagen. Die Wirkweise wurde erklärt mit dem mechanischen Einfluss auf die Frakturzone, der eine Steigerung der Vaskularisation, eine Zunahme der Kallusformation sowie eine Beschleunigung der enchondralen Ossifikation mit sich bringt.

\section{Extrakorporale Stoßwellentherapie}

Auf der Suche nach weiteren komplikationsarmen Alternativen bzw. additiven Möglichkeiten im Sinne einer nichtoperativen Osteoinduktion zur schnelleren Pseudarthrosenheilung wurde in den letzten Jahren neben der elektrischen/elektromagnetischen Stimulation insbesondere die hochenergetische extrakorporale Stoßwellentherapie als Methode genannt, die zumindest in Einzelfällen selbst nach multiplen gescheiterten Voroperationen eine definitive Durchbauung der Pseudarthrosen erbrachte.

$\mathrm{Zu}$ Beginn der 9oer Jahre wurden erste Artikel über die extrakorporale Stoßwellentherapie veröffentlicht, die über die bis dahin etablierte Behandlung von Nierenund Gallensteinen hinausgingen. Valchanou u. Michailov [75], Haist et al. [32] sowie Schleberger u. Senge [65] und Kaulesar Sukul et al. [40] führten die hochenergetische Form der ESWT in die Behandlung von Frakturen mit verzögerter Heilung oder Pseudarthrosenbildung ein und beschrieben dabei Phänomene einer lokalen Dekortikation und Fragmentation mit Stimulation der Osteogenese.

Trotz widersprüchlicher tierexperimenteller Studien, die teils eine Beschleu- nigung, teils eine Verzögerung der Knochenheilung belegten, wurden in den bisher publizierten klinischen Arbeiten hohe Heilungsraten von $75-91 \%$ angegeben [31, $34,64,65,75]$.

\section{Patienten und Methode}

Die hier aufgeführten Ergebnisse wurden bereits veröffentlicht $[59,60]$.

Im Zeitraum von 1992-1998 wurde allen ausgewachsenen Patienten mit einer Pseudarthrose der langen Röhrenknochen der unteren Extremitäten die ESWT als Alternative zur operativen Revision mit Spongiosaplastik angeboten. 20 Frauen und 23 Männer wurden in die prospektive nicht randomisierte Kohortenstudie aufgenommen, sie wiesen ein Durchschnittsalter von 39 Jahren (18-74 Jahren) auf. Im Mittel betrug die Pseudarthrosedauer 13 Monate (9-51 Monate). Die Patienten waren im Durchschnitt 1,9-mal (1-bis 6-mal) voroperiert.17-mal handelte es sich um eine Pseudarthrose nach Fraktur, 26-mal nach Korrekturosteotomie.

Vor der Entscheidung über eine Stoßwellenbehandlung wurden a.-p. und laterale Röntgenaufnahmen, in Ausnahmefällen auch Schrägaufnahmen oder Tomographien, durchgeführt.

\section{Einschluss- und Ausschlusskriterien}

Eingeschlossen wurden nur solche Knochenheilungsstörungen, die seit dem initialen Trauma oder seit der letzten operativen Maßnahme mindestens 9 Monate bestanden.

Ausschlusskriterien waren:

- instabile Situation (Auslockerung der Osteosynthese)

- Pseudarthrosenspalt $>0,5 \mathrm{~cm}$

- Entzündungen (auch Hepatitis, HIV)

- epiphysenfugennahe Defekte im Wachstumsalter

- Trümmerfrakturen

- pathologische Frakturen

- Veränderung des bereits bestehenden Behandlungskonzepts bezüglich Belastung und Ruhigstellung

- medikamentöse Therapie mit Antikoagulanzien, NSAR, Biphosphonaten, Kalziumantagonisten, Immunsuppressiva 

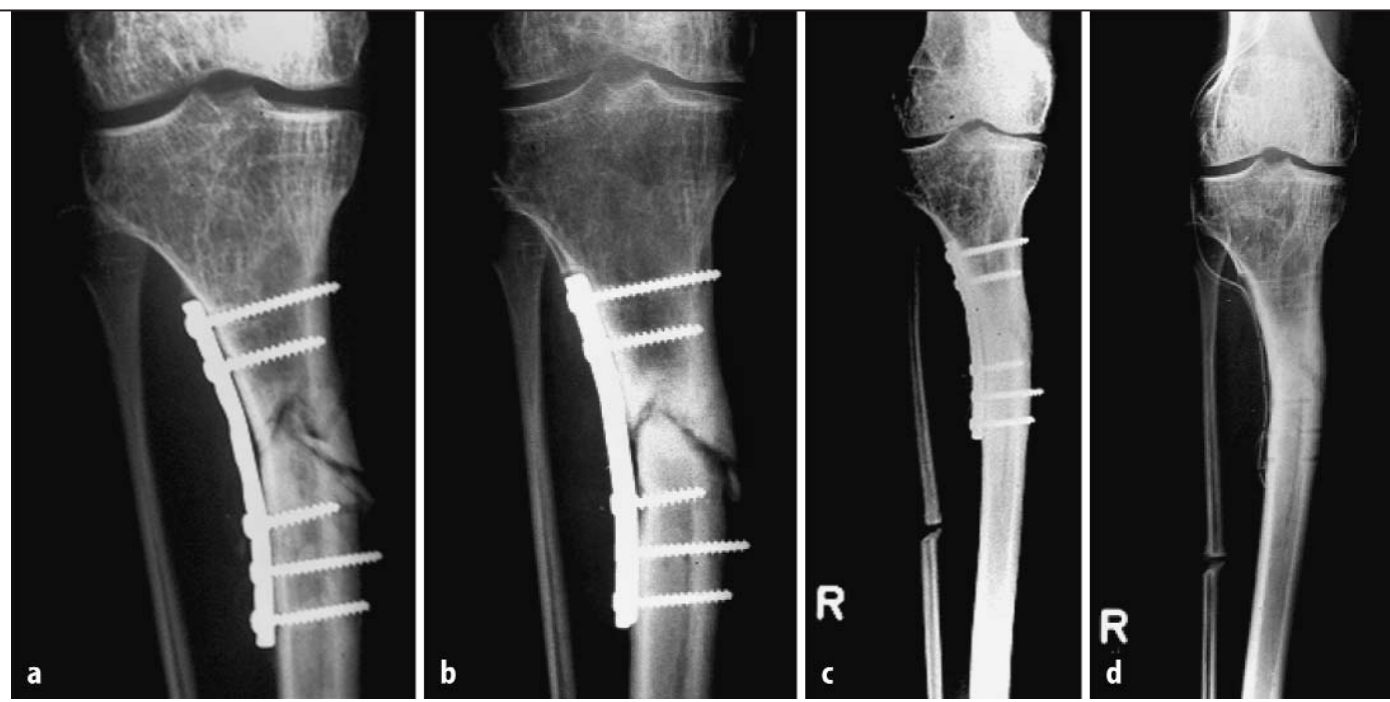

Abb. 1a-d $<$ Patient 12, a 3 Monate nach Pendelosteotomie und Plattenfixation, b 18 Monate nach Osteosynthese, c 4 Monate nach hochenergetischer ESWT knöcherne Heilung, $\mathrm{d}$ Metallentfernung 1 Jahr später

— arterielle oder venöse Durchblutungsstörungen

- Schwangerschaft

- Tumorleiden

\section{Szintigraphie}

Nachdem die Patienten ihre Zustimmung für die ESWT gegeben hatten, wurde in allen Fällen eine regionale ${ }^{99 m}$ Technetiumdicarboxyphosphonat $\left({ }^{99} \mathrm{~m}\right.$ TcDCP)-2-Phasen-Knochenszintigraphie mit einer Picker Dyna Camera 4 durchgeführt, um aktive und inaktive Pseudarthrosen zu unterscheiden. Die ipsilaterale und die kontralaterale Seite wurden miteinander verglichen. Die Auswertung der Blutpoolphase startete 2 min nach der Applikation von $550 \mathrm{MBq}{ }^{99 \mathrm{~m}} \mathrm{TcDCP}$, die Auswertung der Mineralisationsphase begann $3 \mathrm{~h}$ nach der Nuklidgabe. Die Tracerakkumulation wurde von Kollegen der Nuklearmedizin entweder als gering oder nicht (inaktive Pseudarthrose) oder als deutlich (Hyperämie, Hypermineralisation: aktive Pseudarthrose) eingestuft.

\section{Behandlungsverfahren}

Die extrakorporale Stoßwellentherapie wurde innerhalb von 14 Tagen nach der Knochenszintigraphie mit dem Experimentalgerät Siemens Osteostar durchgeführt: In den C-Bogen eines Bildwandlergeräts ist eine Stoßwellenquelle integriert, deren Fokus im Isozentrum des C-Bogens liegt. Das elektromagnetische Stoßwellensystem besteht aus 3 Hauptkomponenten,
- einer akustischen Quelle mit Hochspannungsentladungskreis,

- einem Fokussierelement sowie

- einem Übertragungsmedium, das sich sowohl zwischen Quelle und Linse als auch zwischen Linse und Ankoppelbalg befindet.

Durch eine Kondensatorentladung auf eine Flachspule entsteht ein Stromimpuls, der in der räumlich benachbarten Metallmembran einen Wirbelstrom induziert. Dieser führt zur Abstoßung der Membran von der Spule, was einen akustischen Impuls in der dahinter liegenden Wassersäule erzeugt. Die akustische Welle steilt sich auf ihrem Weg über die schalloptische Linse zur Stoßwelle auf und besitzt im Fokus ihre größte Wirkung. An der Grenzfläche zweier Medien mit ausgeprägt unterschiedlichen Schallleitungseigenschaften, z. B. zwischen Flüssigkeit und Knochen, wird die akustische Energie schließlich in lokale Zug- und Dehnungskräfte umgewandelt.

Der Eingriff erfolgte bei allen Patienten unter stationären Bedingungen in Regionalanästhesie (3-in-1-Block, Plexus- oder Periduralanästhesie). Nach Lokalisierung des zu behandelnden Pseudarthrosebezirks im Isozentrum des Bildwandlers wurde unter Verwendung konventionellen Ultraschallgels die Wasservorlaufstrecke des Stoßwellengenerators angekoppelt.

Danach erfolgte unter Beachtung der topographischen Anatomie die einmalige Applikation von 3000 Impulsen der Energiestufe 9, entsprechend einer Ener- giedichte von $0,6 \mathrm{~mJ} / \mathrm{mm}^{2}$. Die Position des Stoßwellenapplikators wurde in allen Fällen zur Minimierung lokaler Komplikationen und zur Überprüfung der korrekten Position 3- bis 5-mal verändert. Dabei wurden die proximale und die distale Kortikalis als Zielfeld erfasst, es wurde aber auch schräg in den Spalt geschossen. Die Behandlungsdauer variierte zwischen 50 und $75 \mathrm{~min}$.

\section{Statistik}

Nach Rücksprache mit dem Institut für Medizinische Statistik und Datenverarbeitung der Johannes-Gutenberg-Universität erfolgte aufgrund der kleinen Fallzahl und der fehlenden Kontrollgruppe keine statistische Auswertung.

\section{Ergebnisse}

Klinische und radiologische Kontrolluntersuchungen erfolgten nach 8 Wochen, danach alle weiteren 4 Wochen bis zur Konsolidierung oder bis zum 9. Monat nach der ESWT. Die Patienten wurden sämtlich ohne Ausnahme auf diese Weise erfasst.

Alle Röntgenaufnahmen wurden durch einen unabhängigen Untersucher aus der radiologischen Klinik bewertet, in Unkenntnis der Dauer der Pseudarthrose und des Abstands zur ESWT. Eine Heilung wurde angenommen, wenn in Röntgenaufnahmen in 2 Ebenen, in Zweifelsfällen auch auf Schrägaufnahmen, alle 

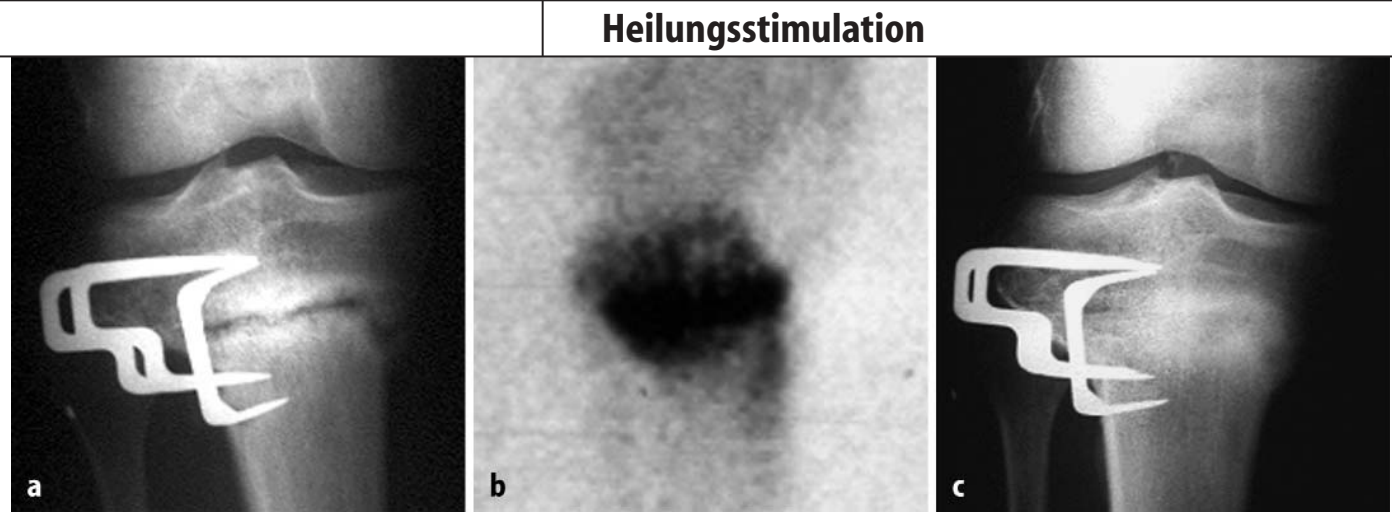

Abb. 2a-c $<$ Patient 32, a a.-p.-Röntgen 13 Monate nach korrigierender Valgisationsosteotomie bei Varusgonarthrose, b signifikante Tracerakkumulation in der Szintigraphie (Mineralisationsphase), ( a.-p.-Röntgen 3 Monate nach hochenergetischer ESWT, knöcherner Durchbau
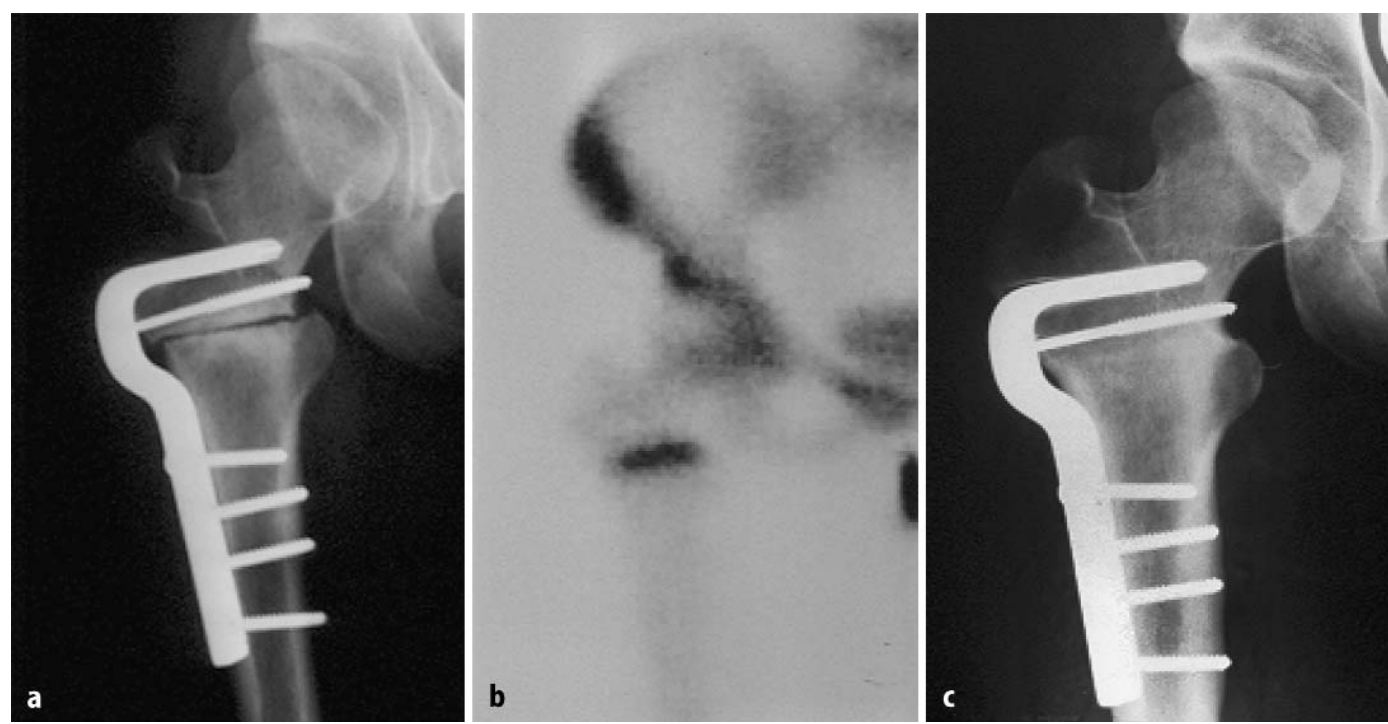

Abb. 3a-c $<$ Patient 42, a a.-p.-Röntgen 15 Monate nach korrigierender intertrochanterer Varisationsosteotomie, b positive Szintigraphie (Mineralisationsphase), ( a.-p.-Röntgen 5 Monate nach hochenergetischer ESWT, knöcherner Durchbau

4 Kortikales überbrückt waren oder kein Spalt in der Tomographie erkennbar war.

Nach durchschnittlich 4,0 $\pm 0,6$ Monaten sahen wir bei 31 von 43 (72,1\%) Pseudarthrosen einen knöchernen Durchbau und gaben die Belastung frei (• Tabelle 1 ;

\section{- Abb. 1,2,3,4).}

17 der $31(54,8 \%)$ erfolgreich mit der ESWT behandelten Patienten hatten szintigraphisch in der Blutpoolphase eine deutliche Anreicherung gezeigt, gegenüber 3 von 12 (25\%) Behandlungsversagern.

25 von $31(80,6 \%)$ erfolgreich behandelten Pseudarthrosen wiesen szintigraphisch eine deutliche Anreicherung in der Mineralisationsphase auf, gegenüber 4 von $12(33,3 \%)$ der Behandlungsversager.

Bei 29 der 35 (82,9\%) Patienten mit einer deutlichen Anreicherung in der Mineralisationsphase kam es nach ESWT zu einer knöchernen Konsolidierung im Vergleich zu 2 von 8 (25\%) Patienten mit einer negativen Szintigraphie. 6 dieser $8 \mathrm{~Pa}$ tienten rauchten mehr als 20 Zigaretten/Tag.
Bis auf lokale Hämatome traten keine Nebenwirkungen nach der hochenergetischen ESWT auf. In keinem Fall kam es zu einer Gefäß- oder Nervenläsion oder zu einer Auslockerung des einliegenden Osteosynthesematerials.

\section{Diskussion}

Beim gepulsten Ultraschall konnte die Knochenneubildung mit einem piezoelektrischen und einem geringgradigen mechanischen Effekt erklärt werden. Eine Steigerung der lokalen Vaskularisation, der Kallusbildung sowie eine beschleunigte enchondrale Ossifikation wurden beschrieben $[21,56,77]$.

Der Wirkmechanismus der Stoßwellen wurde bisher nicht geklärt. Histologische Untersuchungen nach ESWT zeigten an einer Reihe von Tiermodellen eine Stimulation der Osteoneogenese. Quantitative Untersuchungen wurden bis heute nicht publiziert $[18,23,30,47,68]$.

Beunruhigend waren Berichte über eine Störung der Knochenheilung nach
ESWT bei artifiziellen Knochendefekten $[23,37,88]$.

Zum Teil können diese Diskrepanzen durch die fehlende Vergleichbarkeit der zur Verfügung stehenden Stoßwellengeräte erklärt werden. Erst seit 2 Jahren stehen standardisierte Hydrophonmessungen der aktuellen Gerätegeneration zur Verfügung. Ein weiterer Grund für die unterschiedlichen Ergebnisse ist in der Verwendung verschiedener Tiermodelle zu sehen (Hunde, Schafe, Kaninchen), bei denen zusätzlich unterschiedliche Defektund Stabilisierungsformen zur Anwendung kamen.

Anders also als beim gepulsten Ultraschall, wo exzellente klinische Studien eine Beschleunigung der Knochenneubildung bei verzögerter Knochenheilung eindeutig nachgewiesen haben $[24,35,42,86]$, sind alle bisher publizierten Studien zur ESWT vom Studiendesign her unzureichend.

Erste Ergebnisse wurden von Valchanou u. Michailov [75] publiziert, die mit der Methode 1988 begonnen hatten. Von 82 Fällen konnten 70 erfolgreich behandelt wer- 

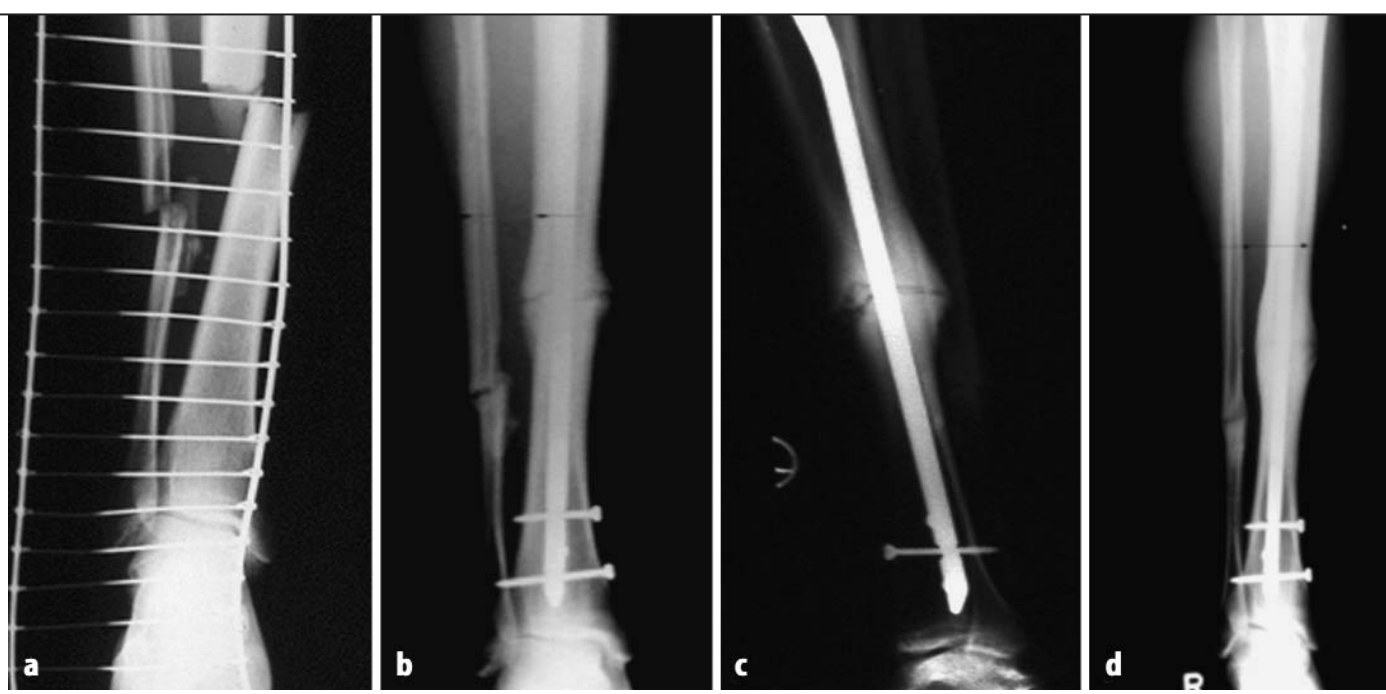

Abb. 4a-d $<$ Patient 43, a a.-p.-Röntgenbild einer geschlossenen Unterschenkelfraktur, b a.-p., c seitliches Röntgenbild 11 Monate nach intramedullarer Nagelversorgung, d a.-p.-Röntgen 4 Monate nach hochenergetischer ESWT, knöcherner Durchbau

den, jedoch fehlen Angaben zur Anamnese, Begleitbehandlungen und Followup. Bürger et al. [12] berichteten eine deutlich geringere Erfolgsquote von 35\% ihrer 37 Patienten. Die Stoßwellentherapie wurde nicht standardisiert durchgeführt, ebenso wenig in den Untersuchungen von Haupt [33] und Diesch u. Haupt [19], die über eine knöcherne Konsolidierung von $76 \%$ und $66 \%$ ihrer 30 bzw. 172 Patienten mit einer Pseudarthrose berichteten. Wirsching et al. [81] behandelten 115 Patienten mit einer Pseudarthrose, durchschnittlich 34 Monate nach der letzten Operation. Mit dem Siemens Osteostar applizierten sie 500 Impulse $/ 5 \mathrm{~mm}$ des Pseudarthrosenspalts mit einer Energieflussdichte von $0,84 \mathrm{~mJ} / \mathrm{mm}^{2}$. Die Begleittherapie wurde nicht geändert. $81 \%$ der Pseudarthrosen kamen zur Ausheilung, wobei in Fällen mit unzureichender knöcherner Reaktion die ESWT wiederholt wurde. Von 52 tibialen Pseudarthrosen heilten sämtliche 24 szintigraphisch hypertrophen Fälle aus, jedoch nur 57\% der 28 atrophen Fälle. Rodriguez et al. [58] berichteten über 3 erfolgreich abgeschlossene Behandlungsfälle. Wang [76] therapierte 40 Patienten hochenergetisch. Alle Patienten wurden einmal behandelt: Bei Pseudarthrosen an Femur oder Tibia wurden 6000 Impulse appliziert, am Unterarm 2000 und an den Handwurzelknochen 1000 Impulse. Nach 6 Monaten waren 28 Patienten beschwerdefrei. Der Pseudarthrosenspalt hatte sich von durchschnittlich 4,5 mm auf 1,6 mm vermindert. Gerdesmeyr et al. [29] therapierten prospektiv 25 Patienten 2-malig in 6-wö- chigem Abstand mit 2000 Impulsen der Energieflussdichte $0,5 \mathrm{~mJ} / \mathrm{mm}^{2}$. Innerhalb von 6 Monaten beobachteten sie eine knöcherne Konsolidierung in $64 \%$. Schaden [62] verwies beim AAOS Kongress auf eine Erfolgsquote von $75,4 \%$ bei 49 Pseudarthrosen und $75 \%$ bei 15 infizierten Pseudarthrosen. Beutler et al. [7] überprüften in einer prospektiven nicht randomisierten Kohortenstudie die von Schleberger [64] 1995 berichtete Erfolgsquote von $91 \%$ bei 45 Fällen. Mit dem gleichen Stoßwellengerät wurden ebenfalls 2-malig 2000 Impulse mit einer Ausgangsspannung von $18 \mathrm{kV}$ appliziert. Bei 11 der 27 erfassten Patienten waren mehrere Reosteosynthesen erfolglos geblieben. Insgesamt beobachteten die Autoren bei einem Follow-up von 6 Monaten eine knöcherne Heilung in 11 Fällen (41\%). Diese trat in jedem Fall innerhalb von 3 Monaten ein.

In unserer nicht randomisierten prospektiven Beobachtungsstudie haben wir uns auf Pseudarthrosen von Femur oder Tibia beschränkt, die mindestens 9 Monate postoperativ bestanden, um nicht nur den deutschen [82], sondern auch den weiter reichenden amerikanischen Kriterien [74] zu genügen. Stringente Aus- und Einschlusskriterien kamen zur Anwendung, die Stoßwellentherapie wurde standardisiert von einem Behandler durchgeführt, die Begleittherapie wurde unverändert belassen. Die Entscheidung über einen Behandlungserfolg fiel anhand radiologischer Kriterien durch einen unabhängigen geblindeten Radiologen. Unter diesen Voraussetzungen erzielten wir bei $72 \%$ unserer Patienten einen Erfolg und fanden einen deutlichen Zusammenhang der Knochenheilung mit dem szintigraphischen Ausgangsbefund.Zusätzlich fiel auf, dass 6 von 8 Patienten mit einer inaktiven Pseudarthrose und nachfolgendem Therapieversagen starke Raucher waren. In Kenntnis des negativen Einflusses von Nikotin auf die Knochenheilung [71] empfehlen wir unseren Patienten nun, mit dem Rauchen vor der ESWT aufzuhören.

Abschließend soll auf einige offensichtliche Schwachpunkte unserer Untersuchung eingegangen werden.

- Obwohl wir die strengeren amerikanischen Kriterien der Pseudarthrosendefinition übernommen haben, ist ohne Kontrollgruppe natürlich kritisch zu hinterfragen, ob es nicht bei einigen der von uns behandelten Fälle nicht auch spontan zu einer Ausheilung gekommen wäre. Eine plazebokontrollierte Studie wurde aber von der lokalen Ethikkommission bereits im Vorfeld abgelehnt. Daher mussten wir uns auf die vorgestellte prospektive nichtrandomisierte Kohortenstudie beschränken.

- Bei allem Bemühen, eine möglichst homogene Patientengruppe zu selektieren, können Unterschiede in der Heilungszeit von Pseudarthrosen nach Fraktur bzw. nach Korrekturosteotomie natürlich nicht ausgeschlossen werden.

- Bei der kleinen Patientenzahl haben wir bewusst auf eine statistische Auswertung verzichtet. 
Die zitierten Untersuchungen zur Wirksamkeit der hochenergetischen ESWT lassen mehr Fragen offen als sie beantworten. Die chirurgische Behandlung von Pseudarthrosen stellt also auch in der näheren Zukunft den Goldstandard dar. Bei der hochenergetischen extrakorporalen Stoßwellentherapie handelt es sich zurzeit trotz inzwischen 10-jähriger Erfahrung noch immer um ein klinisch-experimentelles Verfahren. Die bisher publizierten Ergebnisse rechtfertigen $u$. E. dennoch ihren Einsatz in ausgewählten Einzelfällen, insbesondere auch aufgrund der gut dokumentierten Nichtinvasivität und Komplikationsarmut. Durch konsequenten Ausschluss prognostisch ungünstiger Lokalisationen und Sonderformen kann die Erfolgsrate positiv beeinflusst werden.

Wir selbst können seit 1999 den betroffenen Patienten nur noch die operative Revision anbieten, da ein Ersatz für das inzwischen irreparabel geschädigte Experimentalgerät, wegen der Verweigerung der Kostenübernahme der Behandlung durch die gesetzlichen Krankenkassen, von der Verwaltung des Universitätsklinikums abgelehnt wurde.

\section{Korrespondierender Autor Prof.Dr.J.-D. Rompe}

Orthopädische Universitätsklinik Mainz, Langenbeckstraße 1,55131 Mainz,

E-Mail: rompe@mail.uni-mainz.de

Interessenkonflikt: Der korrespondierende Autor versichert, dass keine Verbindungen mit einer Firma, deren Produkt in dem Artikel genannt ist, oder einer Firma, die ein Konkurrenzprodukt vertreibt, bestehen.

\section{Literatur}

1. Ascherl L (1979) Untersuchungsergebnisse über den elektrischen Widerstand am Knochengewebe. In: Hauptverband Gewerbliche BG Bonn (Hrsg) Schriftenreihe BG-Forschungsinstitut für Traumatologie 3. Hauptverband Gewerbliche BG, Bonn

2. Barker AT, Dixon RA, Sharrard WJW et al. (1984) Pulsed magnetic field therapy for tibial non-union. Interim results of a double-blind trial. Lancet 1: 994-996

3. Bassett CAL, Becker RO (1962) Generation of electrical potentials by bone in response to mechanical stress. Science 137: 1063-1075

4. Bassett CA, Pawluk RJ, Pilla AA (1974) Augmentation of bone repair by inductively coupled electromagnetic fields. Science 184: 575-577

5. Bassett CA, Mitchell SN, Gaston SR (1981) Treatment of ununited tibial diaphyseal fractures with pulsing electromagnetic fields. J Bone Joint Surg Am 63-A: 511523
6. Besch L, Bielstein D, Schuckart M et al. (1994) Analyse von 55 posttraumatischen Pseudarthrosen nach Unterschenkelfraktur.Zentralbl Chir 119: 702-705

7. Beutler S, Regel G, Pape HC et al. (1999) Die extrakorporale Stoßwellentherapie (ESWT) in der Behandlung von Pseudarthrosen des Röhrenknochens. Erste Ergebnisse einer prospektiven klinischen Untersuchung. Unfallchirurg 102: 839-847

8. Bhan S, Mehara AK (1993) Percutaneous bone grafting for nonunion and delayed union of fractures of the tibial shaft. Int Orthop 17: 310-312

9. Blümlein H,McDaniel J, Ziegler WJ et al. (1976) Die Beeinflussung der Pseudarthrosenheilung durch magnetische Wechselfelder in vivo. Langenbecks Arch Chir [Suppl] 1976: 281-285

10. Braun W, Rüter A (1996) Frakturheilung. Morphologische und physiologische Gesichtspunkte. Unfallchirurg 99: 59-67

11. Brighton CT, Shaman P, Heppenstall RB et al. (1995) Tibial nonunion treated with direct current, capacitive coupling, or bone graft. Clin Orthop 321: 223-234

12. Bürger RA, Witzsch U, Haist J et al. (1991) Extrakorporale Stoßwellenbehandlung bei Pseudarthrose und aseptischer Knochennekrose. Urologe A 30: 48-49

13. Cattaneo R, Catagni MA, Guerreschi F (1993) Applications of the llizarov method in the humerus. Hand Clin 9: 729-739

14. Conolly JF (1981) Selection, evaluation and indication for electrical stimulation of ununited fractures. Clin Orthop 161: 39-53

15. Damien CJ, Parsons JR (1991) Bone graft and bone graft substitutes. A review of current technology and applications. J Appl Biomech 2: 187-208

16. De Bastiani G, Aldegheri R, Renzi Brivio L (1986) Dynamic axial fixation. A rational alternative for the external fixation of fractures. Int Orthop 10: 95-99

17. Deibert MC, Mcleod BR, Smith SD et al. (1994) Ion resonance electromagnetic field stimulation for fracture healing in rabbits with a fibular osteotomy.J Orthop Res 12: 878-885

18. Delius M, Draenert K, Aldiek Y et al. (1995) Biological effects of shock waves: in vivo effect of high energy pulses on rabbit bone. Ultrasound Med Biol 21: 1219-1225

19. Diesch R, Haupt G (1997) Anwendung der hochenergetischen extrakorporalen Stoßwellentherapie bei Pseudarthrosen. Orthop Prax 33: 470-471

20. Draenert K (1991) Morphologie der Knochenbruchheilung und ihrer Störungen. In: Springorum HW, Katthagen BD (Hrsg) Aktuelle Schwerpunkte der Orthopädie 2. Thieme, Stuttgart New York, S 56-66

21. Duarte LR (1983) The stimulation of bone growth by ultrasound. Arch Orthop Trauma Surg 101: 153-159

22. Einhorn TA (1995) Enhancement of fracture healing J Bone Joint Surg Am 77-A: 940-956

23. Forriol F, Solchaga L, Moreno JL et al. (1994) The effect of shockwaves on mature and healing cortical bone. Int Orthop 18: 325-329

24. Frankel VH, Ryaby JP, Hagenmeyer K et al. (1996) The effects of low-intensity pulsed ultrasound on the healing of fractures. Abstract SICOT 96, Amsterdam

25. Friedenberg ZB, Brighton CT (1966) Bioelectric potentials in bone.J Bone Joint Surg Am 48-A: 915-923

26. Friedenberg ZB, Harlow MC, Brighton CT (1971) Healing of non-union of the medial malleolus by means of direct current: a case report. J Trauma 11: 883-885

27. Frost HM (1984) The ADFR-concept revisited. Calcif Tissue Int 36: 349-358

28. Fukada E, Yasuda I (1957) On the piezoelectric effect of bone.J Phys Soc Jpn 12: 1158-1162

29. Gerdesmeyr L, Bachfischer K, Peters P et al. (1999) The indication of the application of high energetic extracorporeal shock waves in the treatment of pseudarthrosis - clinical and radiological results. 2nd International Congress of the European Society for Musculoskeletal Shockwave Therapy, London, May 1999
30. Graff J, Richter KD, Pastor J (1988) Effect of high energy shock waves on bony tissue. Urol Res 16: 252-258

31. Haist J (1995) Die Osteorestauration via Stoßwellenanwendung. Eine neue Möglichkeit zur Therapie der gestörten knöchernen Konsolidierung. In: Chaussy C, Eisenberger F, Jochum D et al. (Hrsg) Die Stoßwelle Forschung und Klinik. Attempto, Tübingen, S 157-161

32. Haist J, Steeger D, Witzsch U et al. (1992) The extracorporal shockwave therapy in the treatment of disturbed bone union. 7th International Conference on Biomedical Engineering, Singapore, 1992, pp 222-224

33. Haupt $G$ (1997) Use of extracorporeal shock waves in the treatment of pseudarthrosis, tendinopathy and other orthopedic diseases. J Urol 158: 4-11

34. Haupt G, Katzmeier P (1995) Anwendung der hochenergetischen extrakorporalen Stoßwellentherapie bei Pseudarthrosen, Tendinosis calcarea der Schulter und Ansatztendinosen (Fersensporn, Epicondylitis). In: Chaussy C, Eisenberger F, Jochum D et al. (Hrsg) Die Stoßwelle - Forschung und Klinik. Attempto, Tübingen, S 143-146

35. Heckman JD, Ryaby JP, McCabe J et al. (1994) Acceleration of tibial fracture-healing by non-invasive lowintensity pulsed ultrasound. J Bone Joint Surg Am 76-A: 26-34

36. Hulth A (1989) Current concepts of fracture healing. Clin Orthop 249: 265-284

37. Ikeda K, Tomita K, Takayama K (1999) Application of extracorporeal shock wave on bone: preliminary report. J Trauma 47: 946-950

38. Ilizarov GA (1992) Transosseous osteosynthesis - theoretical and clinical aspects of the regeneration and growth of tissue. Springer, Berlin Heidelberg New York

39. James J, Seijn-Myagkaya GL (1986) Death of osteocytes. Electron microscopy after in vitro ischaemia.J Bone Joint Surg Br 68-B: 620-624

40. Kaulesar Sukul DM, Johannes EJ, Pierik E et al. (1992) The effect of high energy shock waves focused on cortical bone: an in vitro study.J Surg Res 53: 46-51

41. Kraus W, Lechner S (1973) Die Regeneration von Knochengewebe mit langsam alternierenden elektrischen und magnetischen Potentialen. Langenbecks Arch Chir 334: 939

42. Kristiansen TK (1990) The effect of low power specifically programmed ultrasound on the healing of fresh fractures using a Colle's model. J Orthop Trauma 4: 227-228

43. Küntscher GBG (1958) The Küntscher method of intramedullary fixation.J Bone Joint Surg Am 40-A: 17-21

44. Kuner EH (1996) Verzögerte Knochenbruchheilung und Pseudarthrosen des Unterschenkels. In: Baumgartl F, Kremer K, Schreiber HW (Hrsg) Spezielle Chirurgie für die Praxis III/2. Thieme, Stuttgart New York

45. Kuner EH, Berwarth H, Lücke SV (1996) Behandlungsprinzipien bei aseptischen Pseudarthrosen. Orthopäde 25: 394-404

46. Lechner $F$ (1994) Beeinflussung der Knochenbildung durch elektromagnetische Potentiale. Langenbecks Arch Chir 337: 631-635

47. McCormack D, Lane H, McElwain J (1996) The osteogenic potential of extracorporeal shock wave therapy: an in vivo study. Irish J Med Sci 165: 20-22

48. McKibbin $B$ (1978) The biology of fracture healing in long bones.J Bone Joint Surg Br 60-B: 150-152

49. Müller M, Schilling T, Minne HW et al. (1991) A systemic acceleratory phenomenon (SAP) accompanies the regional acceleratory phenomenon (RAP) during healing of a bone defect in the rat.J Bone Miner Res 6: 401-410

50. Parnell EJ, Simonis RB (1991) The effect of electrical stimulation in the treatment of non-union of the tibia. J Bone Joint Surg Br 73-B: S178

51. Pennig D (1990) Zu Biologie des Knochens und der Knochenheilung. Unfallchirurg 93: 488-491 
52. Pepper JR, Herbert MA, Anderson JR et al. (1996) Effect of capacititive coupled electrical stimulation on regenerate bone.J Orthop Res 14: 296-302

53. Perren SM (1992) Biomechanische Grundlagen der Frakturbehandlung. Orthopäde 21: 3-10

54. Perren SM, Cordey J (1977) Die Gewebsdifferenzierung in der Frakturheilung. Unfallheilkd 80: 161-164

55. Pienkowski D, Pollack SR, Brighton CT et al. (1994) Lowpower electromagnetic stimulation of osteotomized rabbit fibulae.J Bone Joint Surg Am 76-A: 489-501

56. Pilla AA, Mont MA, Nasser PR et al. (1990) Non-invasive low-intensity pulsed ultrasound accelerates bone healing in the rabbit.J Orthop Trauma 4:246-253

57. Rijal KP, Kashimoto 0, Sakurai M (1994) Effect of capacitively coupled electrical fields on an experimental model of delayed union of fracture.J Orthop Res 12: 262267

58. Rodriguez de Oya R, Sanchez Benitez de Soto J, Garcia Munilla M (1999) Treatment of non-union with extracorporeal shockwaves. 2nd International Congress of the European Society for Musculoskeletal Shockwave Therapy, London, May 1999

59. Rompe JD, Rosendahl T, Schöllner C et al. (2001) Highenergy extracorporeal shock wave treatment of nonunions. Clin Orthop 387: 102-111

60. Rompe JD, RosendahI T, Schöllner C et al. (2001) Lithotripsie bei femoralen oder tibialen Pseudarthrosen. Trauma Berufskrankh [Suppl 1] 3: 73-75

61. Rosson JW, Simonis RB (1992) Locked nailing for nonunion of the tibia.J Bone Joint Surg Br 74-B: 358-361

62. Schaden W (2000) Single application of extracorporeal shock waves in 97 patients with nonunions or delayed healing fractures. Presentation. 67th Annual Meeting of the American Academy of Orthopaedic Surgeons, March, 2000

63. Schatzker J, Waddell J, Stoll JE (1989) The effects of motion on the healing of cancellous bone. Clin Orthop 245 282-287

64. Schleberger R (1995) Anwendung der extrakorporalen Stoßwelle am Stütz- und Bewegungsapparat im mittelenergetischen Bereich. In: Chaussy C, Eisenberger F, Jochum D et al. (Hrsg) Die Stoßwelle - Forschung und Klinik. Attempto, Tübingen, S 166-174

65. Schleberger R, Senge T (1992) Non-invasive treatment of long bone pseudarthrosis by shock waves (ESWL). Arch Orthop Trauma Surg 111: 224-227

66. Schmit-Neuerburg KP, Stürmer KM, Kehr H et al. (1980) Die Wirksamkeit elektromagnetisch induzierten Wechselstroms auf die Einheilung autologer Spongiosatransplantate bei atrophen Schaftpseudarthrosen. Unfallheilkd 83: 195-201

67. Scott G, King JB (1994) A prospective, double-blind tria of electrical, capacitative coupling in the treatment of non-union of long bones.J Bone Joint Surg Am 76-A: 820-826

68. Seemann 0, Rassweiler J, Chvapil M et al. (1992) Effect of low dose shock wave energy on fracture healing: an experimental study. J Endurol 6: 219-223

69. Segmüller G, Cech 0, Bekier A (1969) Die osteogene Aktivität im Bereich der Pseudarthrose langer Röhrenknochen.Z Orthop Ihre Grenzgeb 106: 599

70. Sharrard WJW (1990) A double-blind trial of pulsed electromagnetic fields for delayed union of tibial fractures. J Bone Joint Surg Br 72-B: 347-355

71. Silcox DH 3rd, Daftari T, Boden SD et al. (1995) The effect of nicotine on spinal fusion. Spine 20: 1549-1553

72. Stürmer KM (1996) Pathophysiologie der gestörten Knochenheilung. Orthopäde 25: 386-393

73. Stürmer KM, Kehr H, Schmit-Neuerburg KP (1979) Fördern niederfrequentes Magnetfeld und Wechselspannung die Knochenheilung? Zentralbl Chir 104: 777-790

74. Taylor JC (1992) Delayed union and nonunion of fractures. In: Crenshaw AH (ed) Campbell's operative orthopaedics, vol II. Mosby, St Louis, Chapt 28
75. Valchanou VD, Michailov P (1991) High energy shock waves in the treatment of delayed and nonunion of fractures. Int Orthop 15: 181-184

76. Wang C (1999) Treatment of fracture non-union with shockwave application. 2nd International Congress of the European Society for Musculoskeletal Shockwave Therapy, London, May 1999

77. Wang SJ, Lewallen DG, Bolander ME et al. (1994) Low intensity ultrasound treatment increases strength in a rat femoral fracture model.J Orthop Res 12: 40-47

78. Warren SB, Brooker AF Jr (1992) Intramedullary nailing of tibial nonunions. Clin Orthop 285: 236-243

79. Weber BG (1982) Klassifizierung der Pseudarthrosen nach morphologischen und klinischen Gesichtspunkten. Z Orthop Ihre Grenzgeb 20: 528-531

80. Weber BG, Cech 0 (1973) Pseudarthrosen - Pathophysiologie, Biomechanik, Therapie, Ergebnisse. Huber, Bern Stuttgart Toronto

81. Wirsching RP, Eich W, Misselbeck E (1998) Langzeitergebnisse nach extrakorporaler Stoßwellentherapie bei Pseudarthrosen. Stosswelle 1: 22-26

82. Wirth CJ (1992) Pseudarthrosen. In: Jäger M, Wirth CJ (Hrsg) Praxis der Orthopädie, 2. Aufl. Thieme, Stuttgart New York, S 284-291

83. Wiss DA, Stetson WB (1994) Nonunion of the tibia treated with a reamed intramedullary nail.J Orthop Trauma 8: 189-194

84. Wolff R (1989) Normale und gestörte Knochenheilung. In: Zilch H, Weber U (Hrsg) Orthopädie. deGruyter, Berlin New York, S 49-57

85. Wu CC, Shih CH (1992) Treatment of 84 cases of femoral nonunion. Acta Orthop Scand 63: 57-60

86. Xavier CAM, Duarte LR (1987) Treatment of non-unions by ultrasound stimulation: first clinical applications. Vortrag AAOS, San Francisco

87. Yasuda I (1953) Fundamental aspects of fracture treatment.J Kyoto Med Soc 4: 395-406

88. Yeaman LD, Jerome CP, McCullough DL (1989) Effects of shock waves on the structure and growth of the immature rat epiphysis.J Urol 141: 670-674

89. Younger EM, Chapmann MW (1989) Morbidity at bone graft donor sites.J Orthop Trauma 3: 192-195

90. Zamora-Navas P, Borras-Verdera A, Antelo-Lorenzo R et al.(1995) Electrical stimulation of bone non-union with the presence of a gap. Acta Orthop Belg 61: 169-176

91. Zichner L (1977) Zur Morphologie der Knochenbildung und Knochenheilung unter dem Einfluß von elektrischem Strom. Habilitationsschrift, Johann-WolfgangGoethe-Universität Frankfurt/Main 